\title{
MAGNETIC FIELDS, BREMSSTRAHLUNG AND SYNCHROTRON EMISSION IN THE FLARE OF \\ 24 OCTOBER 1969
}

H. ZIRIN and GAIL PRUSS

Big Bear Observatory, Hale Observatories California Institute of Technology, Carnegie Institute of Washington, D.C., U.S.A.

and

JOAN VORPAHL

Space Sciences Laboratory, University of California, Berkeley, Calif., U.S.A.

(Received 10 February, 1971)

\begin{abstract}
An impulsive flare October 24, 1969 produced two bursts with virtually identical time profiles of $8800 \mathrm{MHz}$ emission and X-rays above $48 \mathrm{keV}$. The two spikes of hard X-rays correspond in time to the times of sharp brightening and expansion in the $\mathrm{H} \alpha$ flare. The first burst was not observed at frequencies below $3000 \mathrm{MHz}$. This cut off is ascribed to plasma cutoff above the lowlying flare.

A model of the flare based on $\mathrm{H} \alpha$ observations at Big Bear shows that the density of electrons with energy above $10 \mathrm{keV}$ is $5 \times 10^{7}$ if the field density is $10^{11}$. The observed radio flux would be produced by this electron distribution with the observed field of $200 \mathrm{G}$. The $\mathrm{H} \alpha$ emission accompanying the hard electron acceleration is presumed due to excitation of the field atoms by the hard electrons.
\end{abstract}

We have already published (Zirin, 1970) a description of the eruptive flare of October 24, 1968, 2100 UT. We now turn to the flare of October 24, 1969, 2112 UT. These flares are actually different and the sunspot groups in which they occurred were very different in magnetic configuration. So the coincidence of position in the Earth's orbit is irrelevant, but amusing.

The October 24, 1969 flare is of interest in that we have high resolution cinematograms from Big Bear Solar Observatory, fine scan magnetograms from Mt. Wilson for the following day, excellent hard X-ray data from UC Berkeley (OGO-5) and radio observations from various sources. These show a detailed correspondence between $\mathrm{H} \alpha$, radio and X-ray events. More important, the flare produced two X-ray bursts of quite different spectra; the contrast between these is most instructive for our picture of emission by energetic electrons.

The flare occurred in a large, well-observed spot group dominated by preceding spots. Figure 1 shows the group in $\mathrm{H} \alpha$ on the morning of the 24th. Magnetic polarities of larger scale features are marked, as deduced from $\mathrm{H} \alpha$ structure and the fine scan magnetogram obtained by Bhatnagar (1971, Figure 1B) on the 25th. There is an area of bright plage north following the big spots: a filament winds north from it. Some time before the flare the filament separated from this region, which assumed bipolar form. The two bright points making up the south part of the flare are on opposite sides of the 


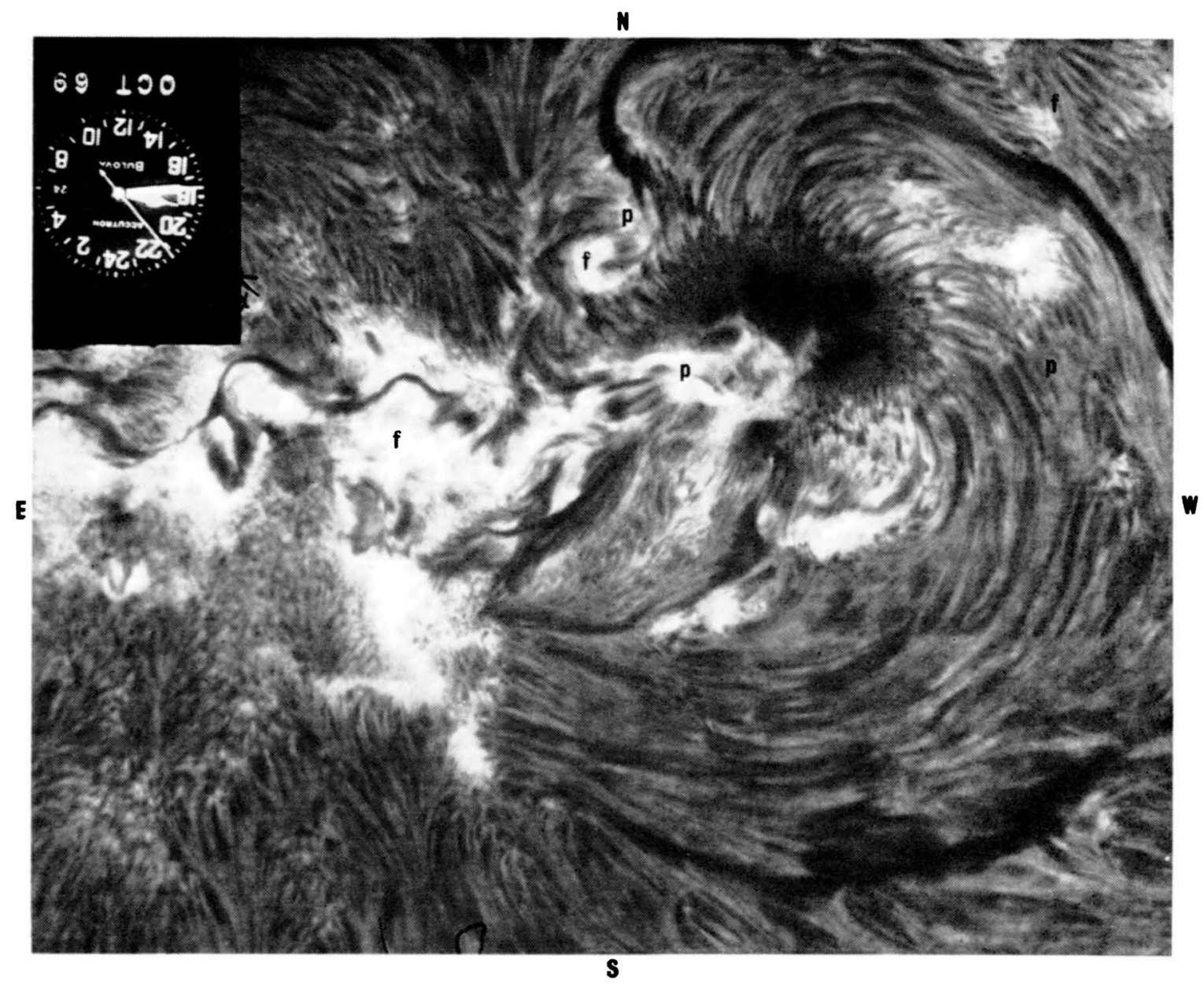

Fig. 1. Ha Big Bear filtergram of the spot group 1838 UT October 24. At the top center we see the small bipolar satellite plage where the flare occurred; the polarity change is marked by a band of transverse dark fibrils in its center. Polarities marked $p$ and $f$.

neutral line in that plage; simultaneously two smaller points brightened under the filament. Unfortunately all of this structure changed after the flare, so the fine scan magnetogram of the 25 th is of limited utility and the field structure must be deduced from the $\mathrm{H} \alpha$ morphology. The structure in the flaring region is very complicated, with a number of small spots; but it is definite that the two bright kernels are on opposite sides of a neutral line. They are not on the neutral line. The flare (Figure 2) began between 21:11:40 and 21:12:00 (unfortunately the interval between frames is $20 \mathrm{~s}$ ) with two points of opposite polarity which rapidly reached maximum brilliance at $21: 12: 40$, accompanied by brightening along the filament neutral line. Growth in these slowed around $21: 13: 30$, followed by a new impulsive development beginning $21: 13: 58$ and continuing to maximum around $21: 16: 00$. This main phase of the flare was marked by outward flow, in contrast to the first phase, which was just brightening in place. The principal movement was outward along the neutral line previously marked by a filament. At the maximum the small bipolar plage as well as the filament channel was all of flare brightness. The flare was a relatively impulsive event as optical 


\section{BIG BEAR SOLAR OBSERVATORY}
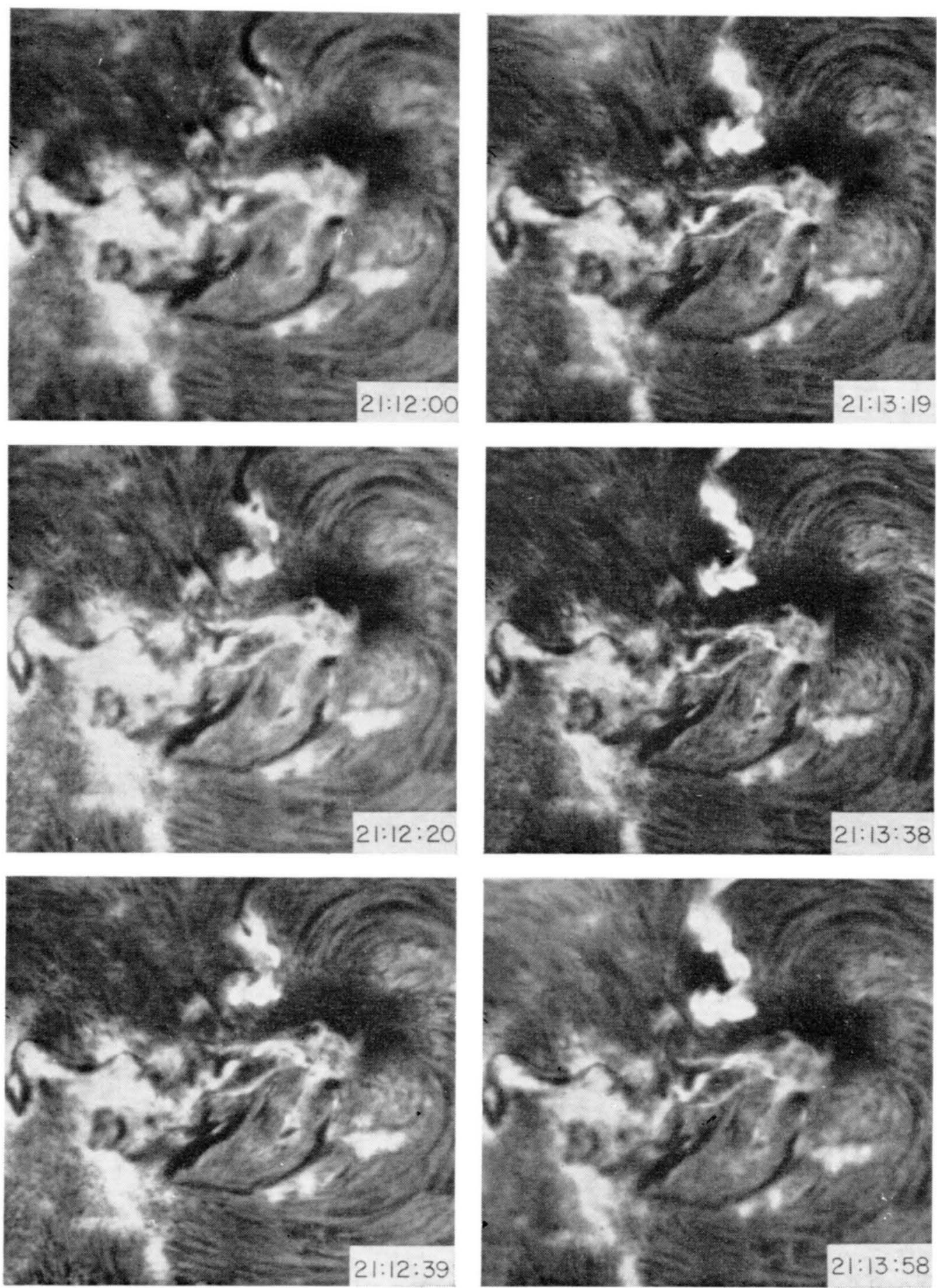

\section{OCTOBER 24, 1969}

Fig. 2. Development of the flare. 
flares go.* Our observations ended just after the flare; the next morning the entire structure was gone.

In Figure 3 we show the X-ray fluxes as measured by the University of California Berkeley group with OGO-5, compared to the radio data from Sagamore Hill. The higher channels show two impulsive bursts, peaking at 21:12:30 and 21:14:00 as well as an extended burst peaking at $21: 15: 00$ in the lowest channel. Because of pile-up of softer photons, there is good reason to identify the low energy maximum with the typical maximum phase soft X-ray burst, such as we identified in the September 11, 1968 event (Vorpahl and Zirin, 1969). The soft X-ray burst (2-12 $\AA$ ) began $21: 12$ and peaked between $21: 15$ and $21: 16$. The peaks of the two impulsive bursts agree perfectly in time with the two fast brightness increases. Measurement of areas also shows steep increases at the time of the impulsive bursts. The spectra of these two bursts have been calculated by Dr Sharad Kane, who finds they correspond to the following differential electron energy spectra:

Burst $1(21: 13)$ :

$$
\frac{\mathrm{d} n_{e}}{\mathrm{~d} E}=E^{-2.21} ; \quad n_{e} n_{i} V=1.8 \times 10^{45}
$$

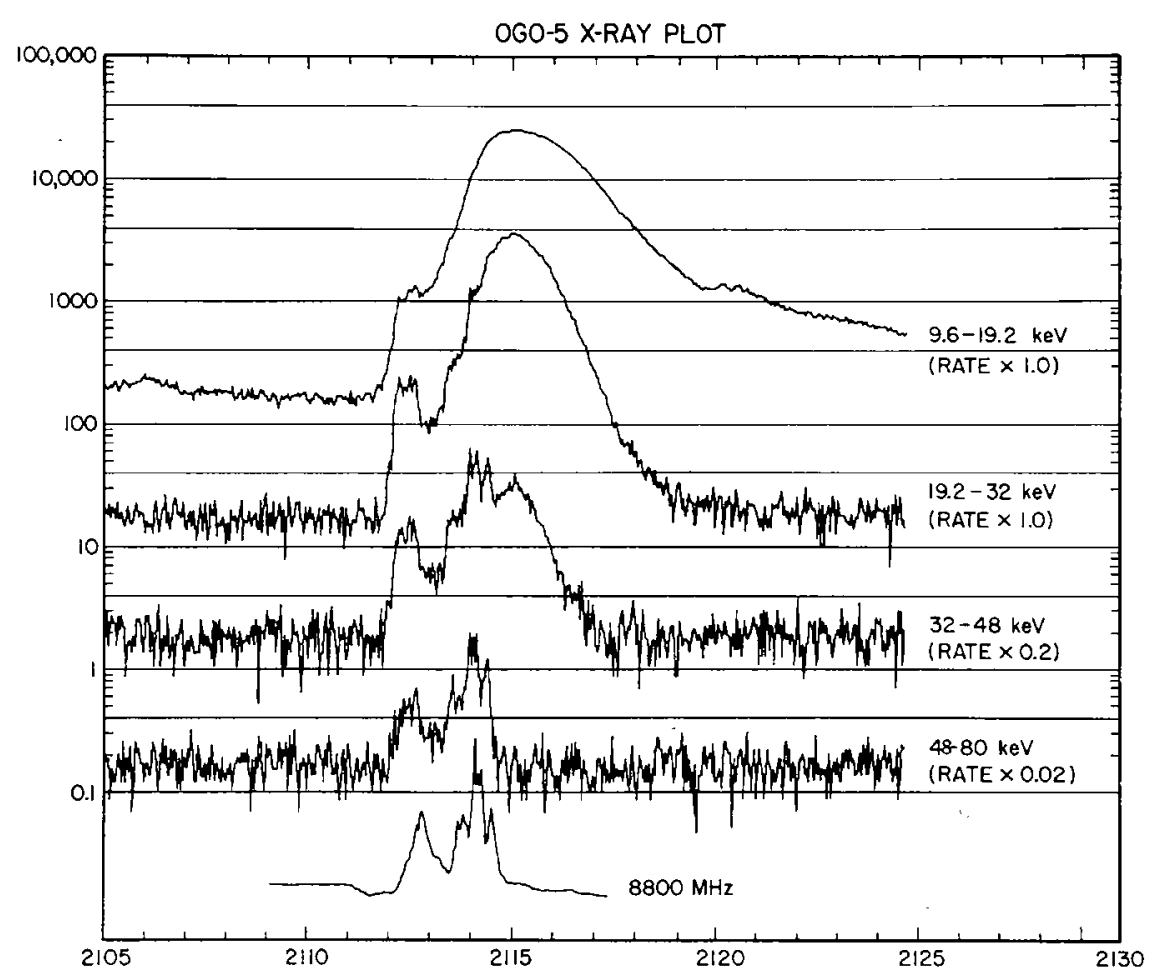

Fig. 3. OGO-V X-ray flux compared to radio flux. We are mostly concerned with the hard burst in the higher energy channels. The great rounded peak at lower energies is mostly due to pile-up from lower energies.

* A. Wood (private communication) reports a sharp increase in the CI continuum (1098 $\AA$ ) measured by the Harvard OSO VI experiment, start $21: 11$, rising to maximum at $21: 14$, with no intermediate dip. This is in line with the simultaneity of XUV and hard X-ray emission noted by Kane and Donnelly (1971). 
Burst $2(21: 14)$

$$
\frac{\mathrm{d} n_{e}}{\mathrm{~d} E}=E^{-3.01} ; \quad n_{e} n_{i} V=1.6 \times 10^{46} .
$$

From these values and the observed area of the flares we can derive the density of energetic electrons. For example, the area $A$ at the peak of the first burst is $2 \times 10^{18} \mathrm{~cm}^{2}$. If we take a thickness $2 \times 10^{8} \mathrm{~cm}$, we have $V=4 \times 10^{26} \mathrm{~cm}^{3}$. For the second flare, which is four times greater in area, and somewhat greater in height, we use $V=3 \times 10^{27}$. Then we have the parameters given in Table I,

TABLE I

Flare parameters

\begin{tabular}{lllllll}
\hline Burst & $V$ & $n_{e} n_{i} V$ & $n_{i}$ & $n_{e}$ & $N_{100}$ & $N_{100} / B A$ \\
\hline I & $4 \times 10^{26}$ & $1.8 \times 10^{45}$ & $10^{10}$ & $5 \times 10^{8}$ & $1.7 \times 10^{34}$ & $5 \times 10^{12}$ \\
& & & $10^{11}$ & $5 \times 10^{7}$ & $1.7 \times 10^{33}$ & $5 \times 10^{13}$ \\
UI & \multirow{2}{*}{$3 \times 10^{27}$} & $1.6 \times 10^{46}$ & $10^{10}$ & $5 \times 10^{8}$ & $1.6 \times 10^{34}$ & $6 \times 10^{11}$ \\
& & & $10^{11}$ & $5 \times 10^{7}$ & $1.7 \times 10^{33}$ & $6 \times 10^{12}$
\end{tabular}

where $N_{100}$ is the total number of electrons with energy $>100 \mathrm{keV}$, obtained by integrating Equation (1) and $N_{100} / B A$ is the Holt-Ramaty (1969) parameter, using $B=200 \mathrm{G}$.

The radio burst as observed at Sagamore Hill is shown in Figure 4. At the higher frequencies it agrees with the X-ray burst in almost every detail. Since this comparison showed slight delay in the radio data, we checked with Dr Castelli, who found that there was in fact no delay; the radio data should be moved forward $10 \mathrm{~s}$, which give perfect agreement. The first radio pulse is cut off at frequencies below $3000 \mathrm{MHz}$, a fact of great importance, which probably tells us the height of the flare in the atmosphere.

There has been considerable discussion concerning the height of flares in the atmosphere. Zirin (1964) described a large explosive flare behind the limb November 20, 1960 that produced a large fadeout with little $\mathrm{H} \alpha$ visible; on the other hand Zirin et al. (1969) showed a perfect correlation between $\mathrm{H} \alpha$ and $\mathrm{X}$-rays in another behind-the-limb event December 2, 1967. Both of these events radiated X-rays from high in the atmosphere; the first was surely a hard event as well. But there is considerable evidence for low-lying flares as well; the hard X-ray burst of September 28, 1961 (Anderson and Winckler, 1962) showed a beautiful example of an impulsive burst at $9500 \mathrm{MHz}$, with no corresponding peak at $3000 \mathrm{MHz}$. It appears that in flares without explosive motion the energetic kernel remains low in the atmosphere, but in explosive events the energetic particles cum magnetic field move outward so that radiation comes from higher levels and no low-frequency radio cutoff appears. If the burst comes from low in the atmosphere, it might be cut off at lower frequencies by overlying absorption (self- 


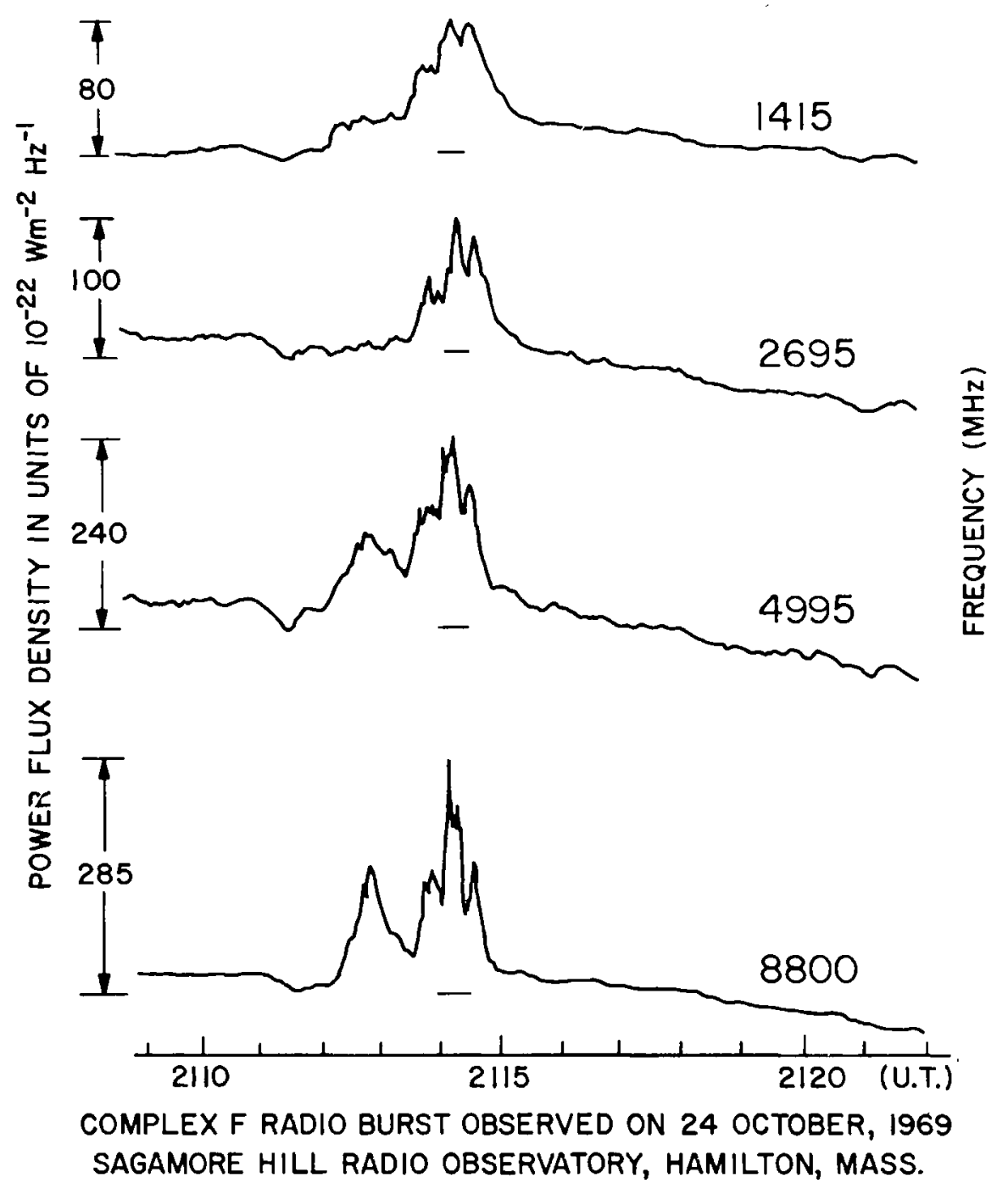

Fig. 4. Radio emission from Sagamore Hill. Times shown should be reduced by $10 \mathrm{~s}$.

absorption is inadequate) or by cutoff at the plasma frequency. If the burst is high in the atmosphere, the cutoff would have to be produced by the Razin effect.

For absorption by coronal material the opacity at $3000 \mathrm{MHz}$ would be $\tau \sim$ $\sim 1.4 \times 10^{-30} n_{e}^{2} L$; thus material at coronal temperatures could produce no appreciable absorption. Plasma at $30000^{\circ}$ would absorb 150 times more effectively; then $\tau \sim 1$ could be achieved with $n_{e} \sim 10^{10}$ and $L \sim 100 \mathrm{~km}$. Alternatively, at $n_{e} \sim 10^{11}$ the plasma frequency is $3000 \mathrm{MHz}$ and all lower frequency emission is cut off. Since we have no good model of an active region we cannot choose between these, but neither a temperature of $30000^{\circ}$ nor a density of $10^{11}$ will exist above $3000 \mathrm{~km}$ in the active region; hence the flare kernel must be below $3000 \mathrm{~km}$ to explain the low frequency cutoff. Since the cutoff is so sharp we believe it is due to the plasma frequency. The fact that the second pulse does not show a low-frequency cutoff and is connected with an outward moving flare supports that view.

Holt and Ramaty (1969) have calculated the gyro-synchrotron emission from a power law electron energy distribution. The peak radio emission occurs typically 
around $15 v_{B}$, where $v_{B}$ is the gyro-frequency, $2.8 \mathrm{MHz} / \mathrm{G}$. Thus for our case, where the peak frequency is $8800 \mathrm{MHz}$ or greater, the field would be around $200 \mathrm{G}$, exactly what we observe on the magnetogram. At higher frequencies the emission falls off as $v^{(1-K) / 2}$ where $K$ is the exponent in the differential electron energy spectrum; in our case the first burst will fall off as $v^{-1 / 2}$. At the lower frequencies the burst is cut off by self-absorption, or if $v_{B}<v_{P}$, by the Razin effect. The condition for a sharp cutoff by the Razin effect is

$$
\alpha \gamma=\frac{3}{2} \frac{v_{B} \gamma}{v_{P}}=\frac{314 B \gamma}{\sqrt{n_{e}}}<1 \quad \gamma=\frac{1}{\sqrt{1-\frac{v^{2}}{c^{2}}}}
$$

In our case, $B=200 \mathrm{G}$, so $\alpha$ is 0.2 for $n_{e}=10^{11}$ and 0.6 for $n_{e}=10^{10}$. The value of $\gamma$ of course varies with the electron energies responsible for any given frequency; this is taken account of in the Holt and Ramaty calculations, but we can use $\gamma=3$ as a rough value. Thus for the observed magnetic field the Razin cutoff takes place for densities similar to those required for plasma cutoff; again the source must lie low in the atmosphere. We cannot use lower values of $B$ to reduce $\alpha$, because then the peak emission would occur at lower frequencies.

It is instructive to examine the applicable curves of Holt and Ramaty (their Figure 1) using the values for $N_{100} / B A$ (which they call $N / B A$ ) from Table 1 . The value for $n_{i}=10^{11}$ (extrapolating to $\alpha=0.2$ ) gives a peak at $5600 \mathrm{MHz}$ of $100 \mathrm{sfu}$; with $n_{i}=10^{10}$ we find a higher peak around $9000 \mathrm{MHz}$ with $3000 \mathrm{sfu}$, about 15 times too much flux.* The latter case also gives too slow a falloff to lower frequencies. What if the burst is higher in the atmosphere, where density is lower? Then $\alpha$ is large and there is no cutoff; there also is too much radio emission because $N_{100}$ becomes large. If we decrease $B$, then the maximum wavelength of the radio emission drops lower. Clearly the best solution is reached with $n_{i}=10^{11}$. But in that case plasma cutoff cuts out the radiation below $3000 \mathrm{MHz}$ and we don't need the Razin effect.

The Holt-Ramaty spectra, even if they give the right flux, are too steeply peaked to correspond to what is observed in most bursts (the reader will convince himself of this by simply glancing at recorded bursts in Solar-Geophysical Data or other publications; microwave bursts often show flat curves between 5000 and $30000 \mathrm{MHz}$, with peak values usually at the higher frequencies for energetic events). Since the Holt-Ramaty calculations are presumably correct, we can only assume that the magnetic field strength and particle spectrum are not uniform across the region. Although the magnetogram does not reveal such small field changes in the bright points that make up the flare, it cannot be ruled out, in view of the inhomogeneity of the $\mathrm{H} \alpha$ emission, and is in fact likely.

O'Dell and Sartori (1970) have recently called attention to a low-frequency cutoff due to the pitch-angle distribution of the electrons. The low frequency slope of this

* Frost (1969) has shown that the hard X-ray spectrum falls off above $100 \mathrm{keV}$ from the normal power spectrum. The reduction of the radio emission might be a factor 3 or so, less than our uncertainty. 
cutoff is $v^{K}$. However our burst falls from $100 \mathrm{sfu}$ to nothing within a factor 2 of frequency; again, plasma cutoff seems to be the only answer.

The second radio spike shows no cutoff at low frequencies. Since it appears to fly out from the region, it must be supposed that it reaches higher elevations, where plasma cutoff is no longer effective; the magnetic field, hence $\alpha$, is smaller here and surely $B$ decreases faster than $n_{e}$. A Razin cutoff, if effective in the first spike, would be even stronger in the second. Plasma cutoff seems a better answer.

The referee of Solar Phys. has raised the question whether anything could be learned about relative densities from the fact that the first burst decays slightly more slowly than the second; even if this difference is small, the second is definitely made up of several individual, short-lived spikes with very fast decay rates. If decay time were proportional to density, this would imply a higher density for the second burst, but it was pointed out by Zirin et al. (1969) that decay by coulomb interaction will lead to a hardening of the spectrum with time, whereas the little information that we have suggests a softening. Calculations of the decay time for various models (Lin and Hudson, 1971) indicate very short relaxation times, but the mechanisms are not well understood. The rise may reflect the time variation of the production mechanism, but the relaxation is due to collision or to escape of particles. In the 2 nd burst, we could guess that the electrons escape more easily in the second flare because of the open field lines, whereas the dipole structure in the first burst confined the electrons better.

Lin and Hudson reject the escape mechanism because they only see $10^{-3}$ of the electrons in interplanetary space at the Earth; but they do not consider at all the possibility of escape to other regions of the Sun such as the photosphere or surrounding cool chromosphere where they lose their energy almost instantly. To be sure, some of this energy appears in the hard X-ray spectrum, but a relativistic electron impinging on the photosphere goes very deep in $1 \mathrm{~s}$, and little of this energy will come back out.

We must remember that all field lines are connected to the photosphere, and losses downward along these lines are very probable, particularly for the most energetic electrons. Indeed this is probably the source of the $\mathrm{H} \alpha$ and XUV emission.

If we consider the short decay time indicated by the spike events and by the LinHudson calculation, then the total number of energetic electrons produced must be multiplied by the ratio of the burst duration to the decay time, at least a factor 5 . The total number of electrons with energy above $9 \mathrm{keV}$ produced in the flare may then be as high as $10^{36}$, or $10^{37}$ in the second burst, corresponding to $10^{29} \mathrm{erg}$. It seems reasonable that much of this energy comes out in the brilliant $\mathrm{H} \alpha$ kernel; but we cannot say that this energetic electron supply is the main source of flare energy because there are plenty of flares without non-thermal X-rays. It suffices to say that when non-thermal electrons are produced, they also produce $\mathrm{H} \alpha$ emission and XUV emission (Kane and Donnelly, 1971) as part of the loss process.

We have explained the characteristics of our flare as those of a low lying energy release at the surface. But what of those flares that have been proven, since they come from spots behind the limb, to come from 20000 or $30000 \mathrm{~km}$ high? Good examples are November 20, 1960 (Zirin, 1964) and March 30, 1970 (Kane and Donnelly, 1970) 
The latter even showed a peak at at least $8800 \mathrm{MHz}$, implying that it carried a field of $200 \mathrm{G}$ up to this high level in the atmosphere. Clearly these are huge events which do not show low frequency cutoffs. The typical flare with low frequency cutoff does not show such huge eruptions.

There are, in summary, two interesting features in this flare: the beautiful correlation between $\mathrm{H} \alpha, \mathrm{X}$-ray, and radio emission; and the juxtaposition of two hard bursts in the same flare, one with a low frequency cutoff and one without. From the first we may conclude that the same electrons are responsible for the radio and $\mathrm{X}$-ray emission, and either the same electrons or the accelerating process are responsible for the $\mathrm{H} \alpha$ emission and the energetic electrons. Of course it has been pointed out many times that $\mathrm{H} \alpha$ is typically radiated at 10000 or $20000 \mathrm{deg}$, whereas the energetic electrons are of course at far greater 'temperatures'. But as we saw, the energetic electrons make up less than $1 \%$ of the total electrons, hence $99 \%$ of the material may be at a lower temperature. Therefore, it is perfectly reasonable that the $\mathrm{H} \alpha$ emission is produced by excitation of atoms of neutral hydrogen in the flare. Indeed, we are most fortunate that we have such a fine tracer of the morphology of the X-ray flare. From the second point we conclude that the low frequency cutoff of some bursts is due to plasma frequency absorption.

It remains to explain why the hard electrons are so well correlated with the extremely bright kernels of $\mathrm{H} \alpha$ emission in flares (Vorpahl and Zirin, 1969). We can only guess that the great brightness results from high density or compression, and only in these cases is the field strong enough to produce the harder electrons. Since the $\mathrm{H} \alpha$ emission from the bright kernels is only $10^{24} \mathrm{erg} / \mathrm{s}$, the energetic electrons along with those below $10 \mathrm{keV}$ could provide the excitation of $\mathrm{H} \alpha$, as well as enough excess thermal energy for the subsequent emission after the electrons themselves are lost or decelerated.

\section{Acknowledgements}

We wish to thank Dr Kane for the use of his X-ray data and several valuable discussions; Drs Castelli, van Allen, and Bhatnagar for their data, and Dr Allan Moffet for several discussions on the question of gyro-synchrotron emission. The Caltech group was supported by NASA under grant NGR 05002034 and NSF under grant GA 24015; the Berkeley group by NASA, under contract NAS 5-9094 and grant NSL 05003017.

\section{References}

Anderson, K. and Winckler, J.: 1962, J. Geophys. Res. 67, 4103.

Bhatnagar, A.: 1971, Solar Phys. 16, 40.

Frost, K.: 1969, Astrophys. J. 159, L159.

Holt, Stephen S. and Ramaty, Reuven: 1969, Solar Phys. 8, 119.

Kane, S. R. and Donnelly, R. F.: 1971, Astrophys. J. 164, 151.

Lin, R. and Hudson, H.: 1971, Solar Phys. 17, 412.

O'Dell, S. L. and Sartori, L.: 1970, Astrophys. J. Letters 62, 137.

Vorpahl, J. and Zirin, H.: 1969, Solar Phys. 11, 285.

Zirin, H.: 1964, Astrophys. J. 140, 1216.

Zirin, H.: 1970, ESSA Report UAG-8, 30.

Zirin, H., Ingham, W., Hudson, H., and McKenzie, D.: 1969, Solar Phys. 9, 269. 